\title{
Non-rigid Deformation Pipeline for Compensation of Superficial Brain Shift
}

Filipe M.M. Marreiros, Sandro Rossitti, Chunliang Wang, and Örjan Smedby

Center for Medical Image Science and Visualization (CMIV), Linköping University, Sweden

Department of Science and Technology (ITN) - Media and Information

Technology (MIT), Linköping University, Sweden

Department of Radiology (IMH), Linköping University, Sweden

\{filipe.marreiros, chunliang. wang, orjan.smedby\}@liu.se

Department of Neurosurgery, County Council of Östergötland, Linköping, Sweden

\{Sandro.Rossitti\}@lio.se

\begin{abstract}
The correct visualization of anatomical structures is a critical component of neurosurgical navigation systems, to guide the surgeon to the areas of interest as well as to avoid brain damage. A major challenge for neuronavigation systems is the brain shift, or deformation of the exposed brain in comparison to preoperative Magnetic Resonance (MR) image sets. In this work paper, a non-rigid deformation pipeline is proposed for brain shift compensation of preoperative imaging datasets using superficial blood vessels as landmarks. The input was preoperative and intraoperative 3D image sets of superficial vessel centerlines. The intraoperative vessels (obtained using 3 Near-Infrared cameras) were registered and aligned with preoperative Magnetic Resonance Angiography vessel centerlines using manual interaction for the rigid transformation and, for the non-rigid transformation, the non-rigid point set registration method Coherent Point Drift. The rigid registration transforms the intraoperative points from the camera coordinate system to the preoperative MR coordinate system, and the non-rigid registration deals with local transformations in the MR coordinate system. Finally, the generation of a new deformed volume is achieved with the Thin-Plate Spline (TPS) method using as control points the matches in the MR coordinate system found in the previous step. The method was tested in a rabbit brain exposed via craniotomy, where deformations were produced by a balloon inserted into the brain. There was a good correlation between the real state of the brain and the deformed volume obtained using the pipeline. Maximum displacements were approximately $4.0 \mathrm{~mm}$ for the exposed brain alone, and $6.7 \mathrm{~mm}$ after balloon inflation.
\end{abstract}

\section{Introduction}

Precise visualization of anatomical structures is an essential component of neurosurgical navigation systems to avoid damage to eloquent cortical brain areas (motor, sensory, visual, and cognitive functions), white matter fiber systems, 
and blood vessels, as well as to guide the surgeon to the region of interest, for example a tumor. A main challenge in neuronavigation systems is the so-called "brain shift", i.e. the deformation of the exposed brain once craniotomy is performed, making the preoperative Magnetic Resonance (MR) image set no longer a geometrically correct map of the area where the operation is carried out. Realtime brain shift correction in imaging datasets is a major challenge in the future development of neuronavigation systems.

In this paper we present a technique to produce a new deformed volume reflecting the deformation required for the preoperative MR volume to correspond to the actual form of the brain, using superficial blood vessels as anatomical landmarks. In previous work [1, we have presented a method to track and obtain 3D points of vessel centerlines. In this work we extend the previous framework and introduce the non-rigid deformation component. The method was evaluated in an animal model (the rabbit brain).

\section{$1.1 \quad$ Related Work}

The problem of brain shift correction has been approached using different landmarks and computer vision systems. D'Apuzzo et al. 2] used a 3D monitoring system to track points in a phantom model of brain shift, with reference points painted on the phantom for tracking. In the work by Hsieh et al. 3], points on the brain surface were tracked, but in this case automatically extracted. Methods using manual extraction of brain sulci lines were reported in two studies: DeLorenzo et al. 4. modelled the brain shift problem in a game theoretic formulation using tracked stereo images to extract the sulci lines, and Chui et al. [5] extracted the sulci lines from 3D surfaces of two different subjects, and performed non-rigid registration. Substantial work was performed to track the cortical surface 6]. Some authors also analyze cortical displacements [7] or perform non-rigid registration using intra-operative MR imaging [8]. The most similar works using vessel tracking are by Reinertsen et al. [9] using ultrasound and by Ding et al. [10] using a laser range scanner.

\section{Material and Methods}

The framework for tracking of superficial brain vessels has been described in a previous publication [1. 3D points along the vessels centerlines were obtained for reference. In order to perform a non-rigid volume deformation, the vessel centerlines were also extracted in the preoperative MR images. The blocks of deformation pipeline presented in this work are:

1. Extraction of vessel centerlines points (preoperative MR, intraoperative multi-view near IR images)

2. Rigid registration

3. Non-rigid registration and matching

4. Brain segmentation and deformation 
The methods were tested on a rabbit brain. The experiment is described below followed by the presentation of the pipeline blocks.

\subsection{Animal Experiment}

The experimental and animal handling procedures were carried out with approval of the local Ethical Committee. The experiment was carried out in two New Zealand white rabbits. The rabbit was chosen as subject because the rabbit brain was considered to be large enough for the experiment, and the cerebrovascular anatomy of the rabbit is closely similar to that of the primate [1].

MR imaging was performed with a 3T Philips Ingenia clinical scanner (Philips Medical Systems, Best, The Netherlands) using a circular two elements surface coil, each element with a diameter of $110 \mathrm{~mm}$. The sequence used was a 3D phase-contrast $\mathrm{MR}$ angiography sequence $(\mathrm{TR}=10.7 \mathrm{~ms}$, $\mathrm{TE}=7.9 \mathrm{~ms}$, flip angle $12^{\circ}, 120$ slices of thickness $1.0 \mathrm{~mm}$ with $0.5 \mathrm{~mm}$ overlap, field of view 89 $\mathrm{mm}$, pixel size $0.4 \mathrm{~mm}$, velocity encoding $5 \mathrm{~cm} / \mathrm{s}$ ) after intravenous injection of $0.1 \mathrm{ml}$ per weight of 100 gram tissue of $50 \%$ diluted gadofosveset trisodium 0.25 $\mathrm{mmol} / \mathrm{ml}$ (Vasovist, Bayer Healthcare AG, Berlin, Germany). The morphological images were used for describing anatomy and the angiographic phase-contrast images $(\mathrm{PCA} / \mathrm{M})$ for identifying the vessels.

The rabbit was anesthesized with Hypnorm (fentanyl citrate $0.315 \mathrm{mg} / \mathrm{mL}$ and fluanozone $10 \mathrm{mg} / \mathrm{mL}$ ) $0.3 \mathrm{~mL} / \mathrm{kg}$ i.m. An ear vein was catheterized for intravenous anesthesia, and a dose Midazolam $2.0 \mathrm{mg} / \mathrm{kg}$ was given i.v. Supplementary doses of Hypnorm $0.1 \mathrm{~mL} / \mathrm{kg}$ i.v. were given at intervals of $30-40$ minutes. During the surgical procedure the animal was lying prone, breathing spontaneously. After local injection of Lidocaine the skull bone was exposed and a hemicraniectomy performed on the right side.

Multi-view near infrared (NIR) intraoperative images of the brain were acquired after craniotomy. The brain was deformed by inserting and inflating a small balloon into the brain. The balloon system used was the microcathetermounted latex occlusion balloon Baltacci-B1 with maximum size 6x9 mm (Balt Extrusion, Montmorency, France). After the experiment the rabbit was killed using an anesthesia overdose.

\subsection{Extraction of Vessel Centerlines Points}

The preoperative vessel centerlines were extracted manually with the CTA Plugin of OsiriX (http://www.osirix-viewer.com). The intraoperative multi-view NIR images were used to extract the vessel centerlines with an inflated and non-inflated balloon. The source images used were the same as in our previous communication [1].

\subsection{Rigid Registration}

Commercial neuronavigation systems are usually equipped with methods to perform rigid registration. These make use of fiducial markers and optical tracking 

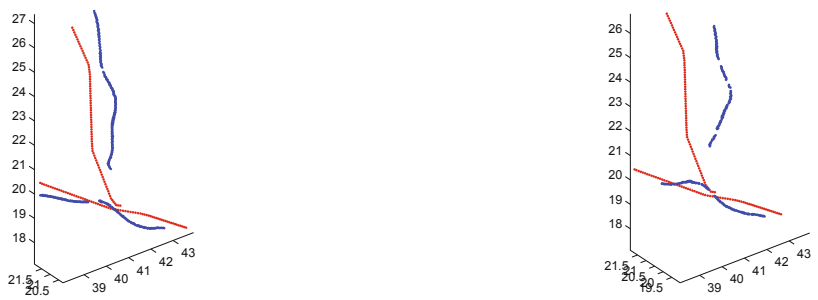

Fig. 1. Manual rigid registration. Intraoperative centerline points of the brain with the balloon uninflated (left) and inflated (right). The intraoperative centerline points were registered to the preoperative coordinate system (blue), the preoperative centerline points (red) suffer no transformation.

systems to rigidly register the current position of the patient's head in the coordinate system of the tracking system to the MR image in its own coordinate system. A drawback is that the fiducials for several reasons can move between MRI acquisition and the operation. In the present experiment, fiducial markers were not used. Instead, a manual transformation was performed using the MeshLab to align the sets of points. The result of the rigid alignment of the intraoperative inflated and non-inflated points to the preoperative MR can be seen in Fig. 1. The same transformation matrix is applied to transform the inflated and non-inflated intraoperative points to an approximation of the preoperative MRI coordinate system. In the ideal case the transformation would align the two coordinate systems perfectly.

\subsection{Non-rigid Registration and Matching}

After rigidly transforming the intraoperative points to the MRI coordinate system, the next step is to register the non-rigid component of the transformation. The method by Myronenko et al. [12, that permits non-rigid registration of 3D points even in the presence of outliers, was used. The Matlab implementation
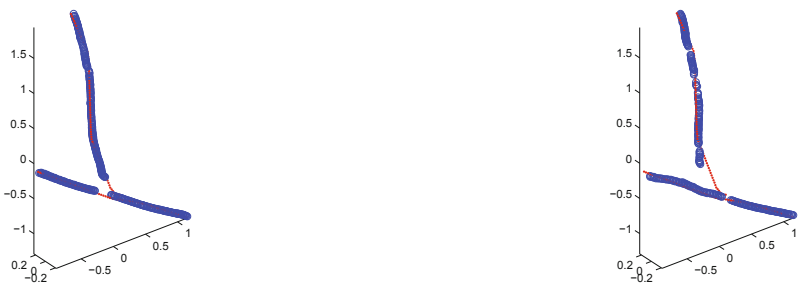

Fig. 2. Non-rigid registration. Intraoperative centerline points of the brain with the balloon uninflated (left) and inflated (right). The intraoperative centerline points (blue) are registered to the preoperative centerline points (red). 

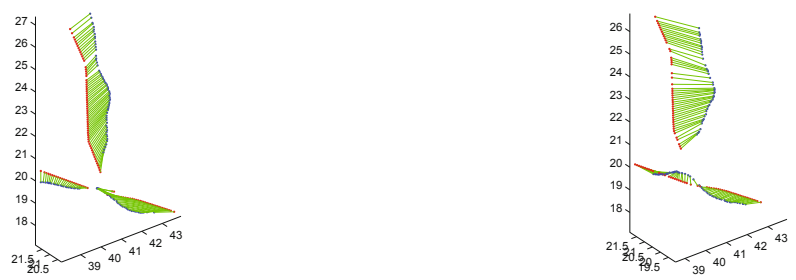

Fig. 3. Centerline point matching. Intraoperative centerline points of the brain with the balloon uninflated (left) and inflated (right). The intraoperative centerline points (blue) are matched to the preoperative centerline points (red). The green lines show the matching between corresponding points.

(https://sites.google.com/site/myronenko/research/cpd) was used with the following non-default parameters: (outliers $=0.8$, beta $=2$, lambda $=10$, $\max$ iter $=100$ ). The input datasets correspond to the preoperative vessel centerline points and the intraoperative vessel centerline points.

After the registration (Fig. 2), the method produces a list of correspondences between the two sets of points. A selection has to be performed to obtain only one-to-one correspondences. If multiple transformed intraoperative points correspond to one preoperative point, the closest one is selected. If the distance between a transformed intraoperative point and its corresponding preoperative point exceeds to $0.2 \mathrm{~mm}$, then this correspondence is discarded. This will discard incorrect matches in the bifurcation area where the intraoperative centerlines are poorly covered. The results can be seen in Fig. 3 .

\subsection{Brain Segmentation and Deformation}

For visualization purposes the brain is segmented using a threshold based level set method reported in 13 . A binary volume mask is generated to exclude regions outside of the brain.

After establishing the correspondence between the preoperative and the intraoperative points rigidly transformed to the preoperative MRI coordinate system, a non-rigid volume deformation method was applied, in this case the thin-plate spline [14 [15] (Fig. 4). In this way, a new deformed volume was created by transforming each new voxel position back to the preoperative MR image and checking the corresponding intensity, a process known as backwards mapping. Since TPS is a global transformation, 30 extra points were added in regions where no deformation is expected to take place.

\section{Results}

The experiment provided surface images of the exposed rabbit brain in a baseline view and after deformation induced by inflation of the balloon inside the brain, that were compared to the imaging acquisitions and virtual reconstructions to 

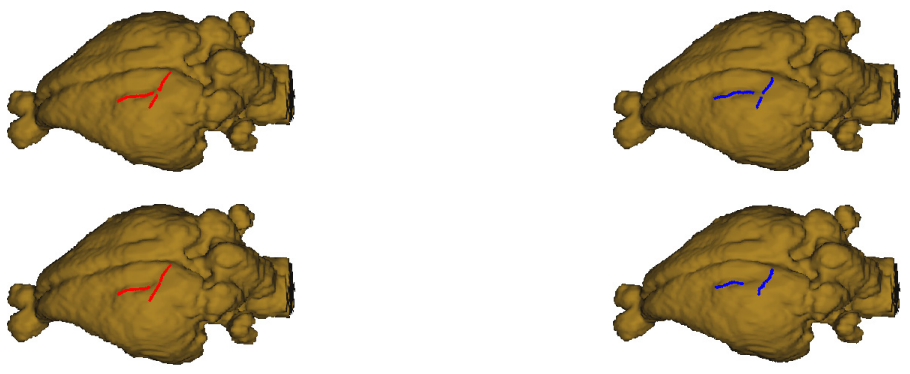

Fig. 4. Brain deformation. Left images: brain before deformation with the selected corresponding points of the preoperative centerlines shown in red. Right images: brain after deformation with the corresponding points of the intraoperative centerlines shown in blue. Top row: uninflated balloon; bottom row: inflated balloon.

evaluate where the main deformations occurred and whether the virtual images were in accordance with the real situation (Fig. 5). The maximum color intensity 255 corresponds to a displacement of $10 \mathrm{~mm}$ and it linearly drops to $0 \mathrm{~mm}$ or no displacement.
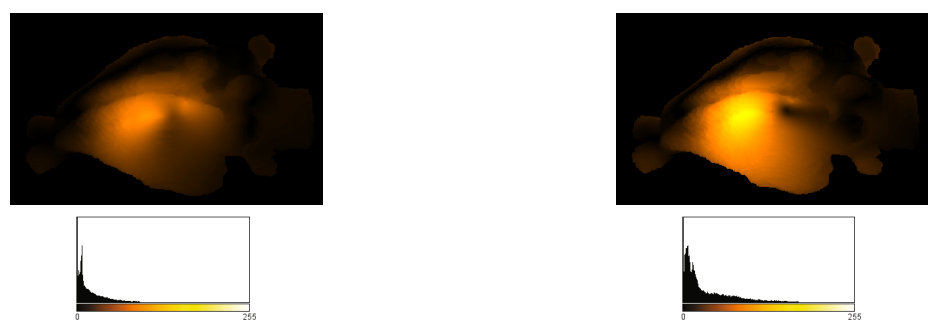

Fig. 5. Surface displacement and histograms with an orange lookup table with the balloon uninflated (left) and inflated (right)

Histograms of these images were generated to quantity the degree of deformation. Analyzing the displacement images one can see a good correlation to the location where the balloon was inserted. Clearly, the deformation is larger in the inflated case. The histogram also illustrates the fact that a larger area was affected by the inflated deformation. In the images with an uninflated balloon, non-zero histogram values were found until 102 or approximately $4.0 \mathrm{~mm}$ and in the inflated case 172 or approximately $6.7 \mathrm{~mm}$. The deformation has an ellipsoid shape (balloon) and maximum displacement constrained by the dimension of the balloon. The maximum value obtained by the inflated case is within the size of the balloon 6x9 mm. Finally, the smoothness of the deformation could also be visualized, with most values concentrated in the lower displacements and slowly decreasing to the larger displacements. 


\section{Discussion}

The present study has introduced a new non-rigid deformation pipeline for compensation of superficial brain shift for neuronavigation purposes, using superficial vessel centerlines points to follow the brain deformation. The method was tested in a rabbit brain where an expansible balloon was inserted. A good correlation was obtained between the current form of the brain and the virtual volume.

Compared to earlier solutions, the present work uses blood vessel centerlines instead of isolated points. This approach resembles the work of Reinertsen et al. [9] using ultrasound and Ding et al. [10] using a laser range scanner. Both use the same non-rigid method that seems to produce similar results to the method selected in this work; a comparison can be found in the article by Myronenko et al. 12 .

In order to guide our deformation method (TPS) we need reliable landmarks with a one-to-one correspondence. Blood vessels centerlines are good candidates because their geometric representation can be robustly matched between preoperative and intraoperative datasets. This process is more prone to errors if we consider the entire surface of the brain (more robust to register lines then surfaces). Even if a good matching is possible, using fine resolution, the number of points used would be much higher making the algorithm slower and possibly run out of memory. The main disadvantage of our method is that it can not compensate the deformation in areas where blood vessels are not present or occluded.

In the ideal case the rigid registration would align the cameras and MRI coordinate systems perfectly. In reality there is a slight misalignment due to several sources of errors. The small misalignments are usually neglected as long as they are within tolerance values.

The preoperative centerline extraction was carried out manually because of the high noise level of the images due to the small brain size. In future studies in humans, an automatic or semi-automatic centerline extraction algorithm should be tested for this purpose.

The proposed method requires further improvements. In particular real-time rigid registration will require the use of fiducial markers. The next stage of our work is to apply this method in neurosurgical operations in human subjects using a commercial neuronavigation system that can track fiducial markers.

Acknowledgments. This work was funded by the Visualization programme of the Swedish Foundation for Strategic Research, the KK Foundation, Vinnova, Invest in Sweden Agency, Vårdalstiftelsen (grant 2009/0079) and the Swedish Childhood Cancer Foundation (grant no. MT2013-0036). The authors are indebted to Johan Kihlberg for assistance with the MRI protocol and to Torbjörn Gustafsson and Per Carleberg for their contribution in the tracking system development. 


\section{References}

1. Marreiros, F., Rossitti, S., Gustafsson, T., Carleberg, P., Smedby, Ö.: Multi-view 3D vessel tracking using near-infrared cameras. In: Proc. of CARS 2013: Image Processing and Visualization (2013)

2. D'Apuzzo, D., Verius, M.: 3D Monitoring of the Intraoperative Brainshift by Means of Photogrammetry. In: Proc. of SPIE-IS\&T Electronic Imaging, vol. 6805 (2008)

3. Hsieh, C., Huang, C., Lee, J., Lee, S., Wu, C.: Estimation of Brain-Surface Deformation with Computer Vision. International Journal of Intelligent Information Processing (IJIIP) 3(1) (2012)

4. DeLorenzo, C., Papademetris, X., Staib, L.H., Vives, K.P., Spencer, D.D., Duncan, J.S.: Image-Guided Intraoperative Cortical Deformation Recovery Using Game Theory: Application to Neocortical Epilepsy Surgery. IEEE Transactions on Medical Imaging 29(2), 322-338 (2010)

5. Chui, H., Rangarajan, A.: A New Algorithm for Non-Rigid Point Matching. In: IEEE Conference on Computer Vision and Pattern Recognition, vol. 2, pp. 2262-2275 (2000)

6. Sun, H., Lunn, K.E., Farid, H., Wu, Z., Roberts, D.W., Hartov, A., Paulsen, K.D.: Stereopsis-Guided Brain Shift Compensation. IEEE Transactions on Medical Imaging 24(8), 1039-1052 (2005)

7. Robers, D.W., Hartov, A., Kennedy, F.E., Miga, M.I., Paulsen, K.D.: Intraoperative Brain Shift and Deformation: A Quantitative Analysis of Cortical Displacement in 28 Cases. Neurosurgery 43(4), 749-758 (1998)

8. Clatz, O., Delingette, H., Talos, I., Golby, A.J., Kikinis, R., Jolesz, F.A., Ayache, N., Warfield, S.K.: Robust Non-Rigid Registration to Capture Brain Shift from Intra-Operative MRI. IEEE Transactions on Medical Imaging 24(11), 1417-1427 (2005)

9. Reinertsen, I., Descoteaux, M., Siddiqi, K., Collins, D.L.: Validation of vessel-based registration for correction of brain shift. Medical Image Analysis 11, 374-388 (2007)

10. Ding, S., Miga, M.I., Noble, J.H., Dumpuri, P., Thompson, R.C., Dawant, B.M.: Semiautomatic registration of pre- and postbrain tumor resection laser range data: method and validation. IEEE Transactions on Biomedical Engineering 56(3), 770-780 (2009)

11. Scremin, O.U., Sonnenschein, R.R., Rubinstein, E.H.: Cerebrovascular anatomy and blood flow measurement in the rabbit. J. Cereb. Blood Flow Metab. 2, 55-66 (1982)

12. Myronenko, A., Song, X., Carreira-Perpiñán, M.Á.: Non-rigid point set registration: Coherent Point Drift. IEEE Transactions on Pattern Analysis and Machine Intelligence 32(12), 2262-2275 (2010)

13. Wang, C., Frimmel, H., Smedby, Ö.: Level-set based vessel segmentation accelerated with periodic monotonic speed function. In: Proc. SPIE 7962, Medical Imaging 2011: Image Processing. Society of Photo-Optical Instrumentation Engineers (2011)

14. Bookstein, F.: Principal warps: Thin-plate splines and the decomposition of deformations. IEEE Transactions on Pattern Analysis and Machine Intelligence 11, 567-585 (1989)

15. Lapeer, R., Prager, R.: 3D shape recovery of a newborn skull using thin-plate splines. Computerized Medical Imaging and Graphics 24, 193-204 (2000) 